F HIAL

KODAI MATH. J.

2 (1979), 300-313

\title{
REPRESENTATION OF ADDITIVE FUNCTIONALS ON VECTOR-VALUED NORMED KÖTHE SPACES
}

\author{
By FuMio HiaI
}

\section{$\S 1$. Introduction.}

Integral representation theory has been developed by many authors for nonlinear additive functionals and operators on measurable function spaces such as Lebesgue spaces and Orlicz spaces; see Alò and Korvin [1], Drewnowski and Orlicz [3-5], Friedman and Katz [6], Martin and Mizel [11], Mizel [12], Mizel and Sundaresan [13-15], Palagallo [16], Sundaresan [19], and Woyczyński [21]. Representation theorems have been obtained also for additive operators on continuous function spaces; see Batt [2] and references therein. The purpose of this paper is to establish representation theorems for additive functionals on Banach space-valued normed Köthe spaces.

In this paper, let $(\Omega, \mathcal{A}, \mu)$ be a $\sigma$-finite measure space and $X$ a real separable Banach space. Let $L_{\rho}(X)$ be an $X$-valued normed Köthe space equipped with an absolutely continuous function norm $\rho$. A functional $\Phi: L_{\rho}(X)$ $\rightarrow \bar{R}$ is called to be additive if $\Phi(f+g)=\Phi(f)+\Phi(g)$ for each $f, g \in L_{\rho}(X)$ such that $\mu(\operatorname{Supp} f \cap \operatorname{Supp} g)=0$. For several types of additive functionals $\Phi: L_{\rho}(X)$

$\rightarrow \bar{R}$, we shall establish integral representations of the form $\Phi(f)=\int_{\Omega} \phi(\omega, f(\omega)) d \mu$ with certain kernel functions $\phi: \Omega \times X \rightarrow \bar{R}$. Representation theorems have been so far obtained for additive functionals which are continuous or rather equicontinuous in some senses. However our method via measurable set-valued functions is applicable to additive lower semicontinuous functionals on $L_{\rho}(X)$.

In $\S 2$, we give definitions and some elementary facts on function norms and normed Köthe spaces. In $\S 3$, a characterization theorem for closed decomposable subsets in $L_{\rho}(X) \times L_{1}$ is established by means of measurable set-valued functions. This characterization will be useful in constructing a set-valued function whose values are closed subsets of $X \times R$ corresponding to epigraphs of an integral kernel function. In $\S 4$, we provide several lemmas on additive functionals and integral functionals on $L_{\rho}(X)$. Finally in $\S 5$, we discuss integral representations for the following cases:

(1) Additive lower semicontinuous functionals on $L_{\rho}(X)$.

Received May 4, 1978 
(2) Additive continuous functionals on $L_{\rho}(X)$.

(3) Bounded linear functionals on $L_{\rho}(X)$.

(4) Additive lower semicontinuous convex functionals on $L_{\rho}(X)$.

The author wishes to express his gratitude to Professor H. Umegakı for his constant encouragement and valuable suggestions.

\section{$\S 2$. Preliminaries.}

Throughout this paper, let $(\Omega, \mathcal{A}, \mu)$ be a fixed $\sigma$-finite measure space and $\bar{A}$ the completion of $\mathcal{A}$ with respect to $\mu$. Let $M^{+}$be the collection of all nonnegative real-valued measurable functions on $\Omega$. A mapping $\rho$ on $M^{+}$to $\bar{R}=[-\infty, \infty]$ is called a function norm if $\rho$ satisfies the following conditions :

(i ) $\rho(\xi) \geqq 0$ and $\rho(\xi)=0$ if and only if $\xi(\omega)=0$ a.e.,

(ii) $\rho(\xi+\zeta) \leqq \rho(\xi)+\rho(\zeta)$,

(iii) $\rho(\alpha \xi)=\alpha \rho(\xi)$ for $\alpha \geqq 0$,

(iv) $\xi(\omega) \leqq \zeta(\omega)$ a. e. implies $\rho(\xi) \leqq \rho(\zeta)$.

Let $\rho$ be a fixed function norm, and let $X$ be a real separable Banach space with dual space $X^{*}$. Note that the notions of strong and weak measurability of functions $f: \Omega \rightarrow X$ are identical, since $X$ is separable. Let $L_{\rho}(X)=$ $L_{\rho}(\Omega, \mathcal{A}, \mu ; X)$ denote the space of all measurable functions $f: \Omega \rightarrow X$ such that $\rho(\|f\|)<\infty$ where $\|f\|=\|f(\cdot)\|$. Then $L_{\rho}(X)$ becomes a normed linear space with the norm $\rho(\|f\|)$ where $\mu$-almost everywhere equal functions are identified. For $X=R$, the space $L_{\rho}=L_{\rho}(R)$ is called a normed Köthe space, and also called a Banach function space if it is complete. Usual $L_{p}(1 \leqq p \leqq \infty)$ spaces and Orlicz spaces are Banach function spaces. The function norm $\rho$ is said to have the Fatou property if $\rho\left(\xi_{n}\right) \uparrow \rho(\xi)$ whenever $\xi_{n} \in M^{+}$and $\xi_{n} \uparrow \xi$, and said to have the weak Fatou property if $\rho(\xi)<\infty$ whenever $\xi_{n} \in M^{+}, \xi_{n} \uparrow \xi$, and $\sup \rho\left(\xi_{n}\right)<\infty$. The weak Fatou property implies the completeness of $L_{\rho}$ and $L_{\rho}(X)$. In this paper, we shall not require $\rho$ to have the weak Fatou property.

The characteristic function of a set $A \in \mathcal{A}$ is denoted by $1_{A}$. A set $A \in \mathcal{A}$ with $\mu(A)>0$ is called unfrendly relative to $\rho$ if $\rho\left(1_{B}\right)=\infty$ for every $B \in \mathcal{A}$ with $B \subset A$ and $\mu(B)>0$. The function norm $\rho$ is called saturated if $\mathcal{A}$ contains no unfriendly sets. There exists a maximal (up to $\mu$-null sets) unfriendly set $\Omega_{\infty}$ and so $\xi(\omega)=0$ a. e. on $\Omega_{\infty}$ for every $\xi \in L_{\rho}$. In order to give representations of additive functionals on $L_{\rho}(X)$, we may assume by removing $\Omega_{\infty}$ from $\Omega$ without loss of generality that $\rho$ is saturated. As a consequence of this assumption, there exists a $\rho$-admissible sequence, i. e., a sequence $\left\{\Omega_{n}\right\}$ in $\mathcal{A}$ with $\Omega_{n} \uparrow \Omega$ such that $\mu\left(\Omega_{n}\right)<\infty$ and $\rho\left(1_{\Omega_{n}}\right)<\infty$ for all $n$. The associate norm $\rho^{\prime}$ is defined by

$$
\rho^{\prime}(\zeta)=\sup \left\{\int_{\Omega} \xi \zeta d \mu: \xi \in M^{+}, \rho(\xi) \leqq 1\right\}, \quad \zeta \in M^{+},
$$


which is also a saturated function norm having the Fatou property.

A function $\xi \in L_{\rho}$ is said to be of absolutely contınuous norm if $\rho\left(1_{A_{n}}|\xi|\right) \downarrow 0$ for every sequence $\left\{A_{n}\right\}$ in $\mathcal{A}$ such that $A_{n} \downarrow \emptyset$. The space $L_{\rho}^{a}$ of all $\xi \in L_{\rho}$ of absolutely continuous norm is a closed order ideal of $L_{\rho}$, that is, $L_{\rho}^{a}$ is a closed subspace of $L_{\rho}$ such that $\zeta \in L_{\rho}^{a}$ and $|\xi(\omega)| \leqq|\zeta(\omega)|$ a. e. imply $\xi \in L_{\rho}^{a}$. Then the dominated convergence theorem holds as follows: If $\xi_{n}(\omega) \rightarrow \xi(\omega)$ a. e. and $\left|\xi_{n}(\omega)\right| \leqq \zeta(\omega)$ a. e. with $\zeta \in L_{\rho}^{a}$, then $\rho\left(\left|\xi_{n}-\xi\right|\right) \rightarrow 0$. We shall always assume that $\rho$ is an absolutely contrnuous norm, i. e., $L_{\rho}^{a}=L_{\rho}$. It is well known that $L_{\rho}^{a}=L_{\rho}$ when $L_{\rho}=L_{p}(1 \leqq p<\infty)$ or more generally when $L_{\rho}$ is an Orlic $z$ space with a Young's function obeying $\triangle_{2}$-condition. After all, it will be assumed in this paper that $\rho$ is a saturated absolutely continuous norm. Therefore the dual space $L_{\rho}{ }^{*}$ of $L_{\rho}$ is isometrically isomorphic to the Banach function space $L_{\rho}$, with the associate norm $\rho^{\prime}$ under the bilinear form $\langle\xi, \zeta\rangle=\int_{\Omega} \xi \zeta d \mu$ of $\xi \in L_{\rho}$ and $\zeta \in L_{\rho^{\prime}}$. For detailed arguments on normed Köthe spaces, see [22, Chap. 15]. The proofs of above stated facts can be found there.

It is worth while remarking that even when $\rho$ is not absolutely continuous, the representation theorems in $\S 5$ hold for additive functionals restricted on $L_{\rho}^{a}(X)=\left\{f \in L_{\rho}(X):\|f\| \in L_{\rho}^{a}\right\}$. However, for the uniqueness of kernel functions, it must be assumed that the carrier of $L_{\rho}^{a}$ (cf. [22, p. 481]) is the whole set $\Omega$. See also Remark 1 to Theorem 5.3.

\section{§3. Decomposable subsets in $\mathrm{L}_{\rho}(X) \times L_{1}$.}

For a set-valued function $F: \Omega \rightarrow 2^{X}$ where $2^{X}$ is the collection of all subsets of $X$, let $D(F)=\{\omega \in \Omega: F(\omega) \neq \emptyset\}$ and $G(F)=\{(\omega, x) \in \Omega \times X: x \in F(\omega)\}$. The inverse image $F^{-1}(U)$ of $U \subset X$ is defined by $F^{-1}(U)=\{\omega \in \Omega: F(\omega) \cap U \neq \emptyset\}$. As to the following conditions for $F: \Omega \rightarrow 2^{X}$ such that $F(\omega)$ is closed for every $\omega \in \Omega$, the implications $(1) \Rightarrow(2) \Leftrightarrow(3) \Rightarrow(4)$ hold, and moreover if $(\Omega, \mathcal{A}, \mu)$ is complete, then all the conditions (1)-(4) are equivalent:

(1) $F^{-1}(C) \in \mathcal{A}$ for every closed subset $C$ of $X$;

(2) $F^{-1}(O) \in \mathcal{A}$ for every open subset $O$ of $X$;

(3) $D(F) \in \mathcal{A}$ and there exists a sequence $\left\{f_{n}\right\}$ of measurable functions $f_{n}: D(F) \rightarrow X$ such that $F(\omega)=\operatorname{cl}\left\{f_{n}(\omega)\right\}$ for all $\omega \in D(F)$;

(4) $G(F) \in A \otimes \mathscr{B}_{X}$ where $\mathscr{B}_{X}$ is the Borel $\sigma$-field of $X$.

A set-valued function $F: \Omega \rightarrow 2^{X}$ is called measurable (resp. weakly measurable) if $F$ satisfies the above condition (1) (resp. (2)). We shall denote by $\mathscr{M}[\Omega ; X]$ the collection of all weakly measurable set-valued functions $F: \Omega \rightarrow 2^{X}$ such that $F(\omega)$ is nonempty and closed for every $\omega \in \Omega$. We observe that if $G(F) \in \mathcal{A} \otimes \mathscr{B}_{X}$ and $F(\omega)$ is nonempty and closed for every $\omega \in \Omega$, then there exists an $F^{\prime} \in \mathscr{M}[\Omega: X]$ such that $F^{\prime}(\omega)=F(\omega)$ a. e. Indeed, since there exists a sequence $\left\{f_{n}\right\}$ of $\overline{\mathcal{A}}$-measurable functions such that $F(\omega)=\operatorname{cl}\left\{f_{n}(\omega)\right\}$ for all $\omega \in \Omega$, we obtain a desired $F^{\prime} \in \mathscr{M}[\Omega ; X]$ by taking $\mathcal{A}$-measurable functions $f_{n}^{\prime}$ with $f_{n}{ }^{\prime}(\omega)=f_{n}(\omega)$ a. e. and defining $F^{\prime}(\omega)=\operatorname{cl}\left\{f_{n}^{\prime}(\omega)\right\}$. For more complete 
discussions of measurability of set-valued functions whose values are closed subsets in a separable metric spaces, see [9] and [20].

Let $M$ be a set of measurable functions $f: \Omega \rightarrow X$. We call $M$ decomposable if $1_{A} f+1_{\Omega \backslash A} g \in M$ for each $f, g \in M$ and $A \in \mathcal{A}$. It is clear that if $M$ is decomposable, then $\sum_{i=1}^{n} 1_{A_{\imath}} f_{i} \in M$ for each finite measurable partition $\left\{A_{1}, \cdots, A_{n}\right\}$ of $\Omega$ and $\left\{f_{1}, \cdots, f_{n}\right\} \subset M$. We showed in [8, Theorem 3.1] that any closed decomposable subset of $L_{p}(X), 1 \leqq p<\infty$, is characterized as a set of the form $S_{p}(F)$ $=\left\{f \in L_{p}(X): f(\omega) \in F(\omega)\right.$ a. e. $\}$ with $F \in \mathscr{M}[\Omega ; X]$. In this section, we obtain an analogous result for subsets of $L_{\rho}(X) \times L_{1}$ which will play an important role in the proof of Theorem 5.1. The product space $L_{\rho}(X) \times L_{1}$ is equipped with the norm $\rho(\|f\|)+\|\xi\|_{1}$ for $f \in L_{\rho}(X)$ and $\xi \in L_{1}$ where $\|\xi\|_{1}$ is the $L_{1}$-norm. A subset $M$ of $L_{\rho}(X) \times L_{1}$ is decomposable if and only if $\left(1_{A} f+1_{\Omega \backslash A} g, 1_{A} \xi+1_{\Omega \backslash A} \zeta\right) \in M$ for each $(f, \xi),(g, \zeta) \in M$ and $A \in \mathcal{A}$. For given $F \in \mathscr{M}[\Omega ; X \times R]$, we define the subset $S_{\rho, 1}(F)$ of $L_{\rho}(X) \times L_{1}$ by

$$
S_{\rho, 1}(F)=\left\{(f, \xi) \in L_{\rho}(X) \times L_{1}:(f(\omega), \xi(\omega)) \in F(\omega) \text { a. e. }\right\} .
$$

We first give some properties of subsets $S_{\rho, 1}(F)$ in the following lemmas.

LEMma 3.1. If $F \in \mathscr{M}[\Omega ; X \times R]$, then $S_{\rho, 1}(F)$ is closed in $L_{\rho}(X) \times L_{1}$.

Proof. Let $\left\{\left(f_{n}, \xi_{n}\right)\right\}$ be a sequence in $S_{\rho, 1}(F)$ convergent to $(f, \xi) \in L_{\rho}(X)$ $\times L_{1}$. Passing to a subsequence, we may assume that $\rho\left(\left\|f_{n}-f\right\|\right)<1 / 2^{n}$ for all $n$ and $\xi_{n}(\omega) \rightarrow \xi(\omega)$ a. e. To prove $(f, \xi) \in S_{\rho, 1}(F)$, it now suffices to show that $\left\|f_{n}(\omega)-f(\omega)\right\| \rightarrow 0$ a.e. Taking a $\rho$-admissible sequence, we may assume in addition that $1_{\Omega} \in L_{\rho}$. For each $k>0$, let $A_{n}=\left\{\omega \in \Omega:\left\|f_{n}(\omega)-f(\omega)\right\| \geqq 1 / k\right\}$ and $A_{\infty}=\bigcap_{m=1}^{\infty} \bigcup_{n=m}^{\infty} A_{n}$. Since $\rho\left(1_{A_{n}}\right) \leqq \rho\left(k\left\|f_{n}-f\right\|\right)<k / 2^{n}$, we have

$$
\rho\left(1_{A_{\infty}}\right) \leqq \sum_{n=m}^{j} \rho\left(1_{A_{n}}\right)+\rho\left(1_{n>j} \cup_{A_{n}}\right)<k / 2^{m-1}+\rho\left(1_{n>>}^{\cup_{A} A_{n}}\right)
$$

for each $j \geqq m \geqq 1$. Since $\rho$ is absolutely continuous, it follows that $\rho\left(1_{n>j} A_{n}\right) \downarrow 0$ as $\jmath \rightarrow \infty$, so that $\rho\left(1_{A_{\infty}}\right)=0$ and hence $\mu\left(A_{\infty}\right)=0$. Letting $k=1,2, \cdots$, we obtain

$$
\mu\left(\bigcup_{k=1}^{\infty} \bigcap_{m=1}^{\infty} \bigcup_{n=m}^{\infty}\left\{\omega \in \Omega:\left\|f_{n}(\omega)-f(\omega)\right\| \geqq 1 / k\right\}\right)=0,
$$

which shows that $\left\|f_{n}(\omega)-f(\omega)\right\| \rightarrow 0$ a. e. Thus the lemma is proved.

LEMMA 3.2. If $F \in \mathscr{M}[\Omega ; X \times R]$ and $S_{\rho, 1}(F)$ is nonempty, then there exists a sequence $\left\{\left(f_{n}, \xi_{n}\right)\right\}$ in $S_{\rho, 1}(F)$ such that $F(\omega)=\operatorname{cl}\left\{\left(f_{n}(\omega), \xi_{n}(\omega)\right)\right\}$ for all $\omega \in \Omega$.

Proof. There exists a sequence $\left\{\left(g_{k}, \zeta_{k}\right)\right\}$ of measurable functions $g_{k}$ : $\Omega \rightarrow X$ and $\zeta_{k}: \Omega \rightarrow R$ such that $F(\omega)=\operatorname{cl}\left\{\left(g_{k}(\omega), \zeta_{k}(\omega)\right)\right\}$ for all $\omega \in \Omega$ (see the above condition (3)). Since $S_{\rho, 1}(F) \neq \emptyset$, we can select an element $(f, \xi) \in S_{\rho, 1}(F)$ such that $(f(\omega), \xi(\omega)) \in F(\omega)$ for all $\omega \in \Omega$. Taking a $\rho$-admissible sequence $\left\{\Omega_{\jmath}\right\}$, we define 


$$
\begin{array}{r}
A_{\jmath m k}=\left\{\omega \in \Omega_{\jmath}: m-1 \leqq\left\|g_{k}(\omega)\right\|+\left|\zeta_{k}(w)\right|<\omega\right\}, \\
f_{\jmath m k}=1_{A_{\jmath m k}} g_{k}+1_{\Omega \backslash A_{\jmath m k}} f, \quad \xi_{\jmath m k}=1_{A_{\jmath m k}} \zeta_{k}+1_{\Omega \backslash A \jmath m k} \xi, \\
j, m, k \geqq 1 .
\end{array}
$$

Then it is easy to see that $\left\{\left(f_{\jmath m k}, \xi_{\jmath m k}\right)\right\} \subset S_{\rho, 1}(F)$ and $F(\omega)=\operatorname{cl}\left\{\left(f_{\jmath m k}(\omega), \xi_{\jmath m k}(\omega)\right)\right\}$ for all $\omega \in \Omega$, completing the proof.

LEMMA 3.3. If $F \in \mathscr{M}[\Omega ; X \times R]$ and $S_{\rho, 1}(F)$ is nonempty and convex, then $F(\omega)$ is convex for a.e. $\omega \in \Omega$.

Proof. By Lemma 3.2, there exists a sequence $\left\{\left(f_{n}, \xi_{n}\right)\right\}$ in $S_{\rho, 1}(F)$ such that $F(\omega)=\operatorname{cl}\left\{\left(f_{n}(\omega), \xi_{n}(\omega)\right)\right\}$ for all $\omega \in \Omega$. Since $\left(\left(f_{i}+f_{j}\right) / 2,\left(\xi_{i}+\xi_{j}\right) / 2\right) \in S_{\rho, 1}(F)$, we can take an $N \in \mathcal{A}$ with $\mu(N)=0$ such that

$$
\left(\left(f_{i}(\omega)+f_{j}(\omega)\right) / 2,\left(\xi_{i}(\omega)+\xi_{j}(\omega)\right) / 2\right) \in F(\omega), \quad i, j \geqq 1, \omega \in \Omega \backslash N .
$$

This shows that $F(\omega)$ is convex for every $\omega \in \Omega \backslash N$, and the lemma is proved.

THEOREM 3.4. Let $M$ be a nonempty subset of $L_{\rho}(X) \times L_{1}$. Then there exists an $F \in \mathscr{M}[\Omega ; X \times R]$ such that $M=S_{\rho, 1}(F)$ if and only if $M$ is closed and decomposable in $L_{\rho}(X) \times L_{1}$.

Proof. If there exists an $F \in \mathscr{M}[\Omega ; X \times R]$ such that $M=S_{\rho, 1}(F)$, then $M$ is closed by Lemma 3.1 and clearly decomposable.

To prove the converse, let $M$ be a nonempty closed and decomposable subset of $L_{\rho}(X) \times L_{1}$. Take an element $\left(f_{0}, \xi_{0}\right) \in M$ and let $M_{0}=\left\{\left(f-f_{0}, \xi-\xi_{0}\right)\right.$ : $(f, \xi) \in M\}$. Then $M_{0}$ is a closed decomposable subset of $L_{\rho}(X) \times L_{1}$ containing $(0,0)$. If there exists an $F_{0} \in \mathscr{M}[\Omega ; X \times R]$ such that $M_{0}=S_{\rho, 1}\left(F_{0}\right)$, then defining $F(\omega)=F_{0}(\omega)+\left(f_{0}(\omega), \xi_{0}(\omega)\right)$ we obtain $F \in \mathscr{M}[\Omega ; X \times R]$ and $M=S_{\rho, 1}(F)$. Thus we may assume that $M$ contains (0,0). Now let $M_{1}=M \cap\left(L_{1}(X) \times L_{1}\right)$ and $M_{2}$ the closure of $M_{1}$ in $L_{1}(X) \times L_{1}$. Then it follows that $M_{2}$ is a nonempty closed and decomposable subset of $L_{1}(X) \times L_{1}$. Noting $L_{1}(X) \times L_{1}=L_{1}(X \times R)$ where the norm of $X \times R$ is taken by $\|(x, \alpha)\|=\|x\|+|\alpha|$, we obtain, by [8, Theorem $3.1]$, an $F \in \mathscr{M}[\Omega ; X \times R]$ such that

$$
M_{2}=\left\{(f, \xi) \in L_{1}(X) \times L_{1}:(f(\omega), \xi(\omega)) \in F(\omega) \text { a. e. }\right\} .
$$

We shall then prove that $M=S_{\rho, 1}(F)$. For each $(f, \xi) \in L_{\rho}(X) \times L_{1}$, taking a $\rho$ admissible sequence $\left\{\Omega_{n}\right\}$ we put $A_{n}=\left\{\omega \in \Omega_{n}:\|f(\omega)\| \leqq n\right\}$ for $n \geqq 1$. Then $\left(1_{A_{n}} f, 1_{A_{n}} \xi\right) \in L_{1}(X) \times L_{1}$ for all $n$ and it follows from $A_{n} \uparrow \Omega$ that

$$
\rho\left(\left\|1_{A_{n}} f-f\right\|\right)+\left\|1_{A_{n}} \xi-\xi\right\|_{1}=\rho\left(1_{\Omega \backslash A_{n}}\|f\|\right)+\left\|1_{\Omega \backslash A_{n}} \xi\right\|_{1} \downarrow 0 .
$$

Thus we deduce in view of $(0,0) \in M$ that $M_{1}$ and $S_{\rho, 1}(F) \cap\left(L_{1}(X) \times L_{1}\right)=$ $S_{\rho, 1}(F) \cap M_{2}$ are dense in $M$ and $S_{\rho, 1}(F)$, respectively. Since both $M$ and $S_{\rho, 1}(F)$ are closed, it remains to show that $M_{1} \subset S_{\rho, 1}(F)$ and $S_{\rho, 1}(F) \cap M_{2} \subset M$. The first 
inclusion is obvious. To see the second inclusion, let $(f, \xi) \in S_{\rho, 1}(F) \cap M_{2}$. Then there exists a sequence $\left\{\left(f_{k}, \xi_{k}\right)\right\}$ in $M_{1}$ convergent in $L_{1}(X) \times L_{1}$ to $(f, \xi)$. It can be assumed that $\left\|f_{k}(\omega)-f(\omega)\right\| \rightarrow 0$ a.e. Taking a $\rho$-admissible sequence $\left\{\Omega_{n}\right\}$, we put $B_{n k}=\left\{\omega \in \Omega_{n}:\left\|f_{k}(\omega)\right\| \leqq\|f(\omega)\|+1\right\}$ for $n, k \geqq 1$. As $k \rightarrow \infty$ for each fixed $n$, it follows from $\mu\left(\Omega_{n} \backslash B_{n k}\right) \rightarrow 0$ that

$$
\left\|1_{B_{n k}} \xi_{k}-1_{\Omega_{n}} \xi\right\|_{1} \leqq\left\|\xi_{k}-\xi\right\|_{1}+\left\|1_{\Omega_{n} \backslash B_{n k}} \xi\right\|_{1} \rightarrow 0 .
$$

Moreover, since

$$
2\|f\|+1_{\Omega_{n}} \geqq\left\|1_{B_{n k}} f_{n}(\omega)-1_{\Omega_{n}} f(\omega)\right\| \rightarrow 0 \text { a. e., }
$$

we obtain $\rho\left(\left\|1_{B_{n k}} f_{k}-1_{\Omega_{n}} f\right\|\right) \rightarrow 0$ by the dominated convergence theorem. Since $\left(1_{B_{n k}} f_{k}, 1_{B_{n}} \xi_{k}\right) \in M$ by $(0,0) \in M$, it follows that $\left(1_{\Omega_{n}} f, 1_{\Omega_{n}} \xi\right) \in M$ for all $n$, so that $(f, \xi) \in M$. Thus $M=S_{\rho, 1}(F)$ is proved.

\section{Additive functionals and integral functionals.}

A functional $\phi: V \rightarrow \bar{R}$ on a topological vector space $V$ is called proper if $\phi(x)>-\infty$ for all $x \in V$ and $\phi \neq \equiv \infty$. The epigraph Epi $\phi$ of $\phi$ is defined by Epi $\phi$ $=\{(x, \alpha) \in V \times R: \phi(x) \leqq \alpha\}$. A functional $\phi: V \rightarrow \bar{R}$ is lower semicontinuous (resp. convex) if and only if Epi $\phi$ is closed (resp. convex) in $V \times R$. Let $\phi$ : $\Omega \times X \rightarrow \bar{R}$ be an $\mathcal{A} \otimes \mathscr{B}_{X}$-measurable function. For a measurable function $f$ : $\Omega \rightarrow X$, since the function $\phi(\omega, f(\omega))$ is measurable, we define $I_{\phi}(f)=$ $\int_{\Omega} \phi(\omega, f(\omega)) d \mu$ if the integral exists permitting $\pm \infty$. We call $I_{\phi}$ the integral functional associated with the kernel function $\phi$. A function $\phi: \Omega \times X \rightarrow \bar{R}$ is called normal if $\phi$ is $A \otimes \mathscr{B}_{X}$-measurable and $\phi(\omega, \cdot)$ is lower semicontinuous for every $\omega \in \Omega$. Let Epi $\phi: \Omega \rightarrow 2^{X \times R}$ be defined by $($ Epi $\phi)(\omega)=\operatorname{Epi} \phi(\omega, \cdot)$. By way of the measurability of the function $(\omega, x, \alpha) \mapsto \phi(\omega, x)-\alpha$ with respect to $A \otimes \mathscr{B}_{X \times R}=A \otimes \mathscr{B}_{X} \otimes \mathcal{B}_{R}$, it is seen that $\phi$ is normal if and only if $G($ Epi $\phi)$ $\in \mathcal{A} \otimes \mathscr{B}_{X \times R}$ and (Epi $\left.\phi\right)(\omega)$ is closed for every $\omega \in \Omega$. Thus $\phi$ is normal if Epi $\phi$ $\in \mathscr{M}[\Omega ; X \times R]$, and vice versa when $(\Omega, \mathcal{A}, \mu)$ is complete.

For a measurable function $f: \Omega \rightarrow X$, let Supp $f=\{\omega \in \Omega: f(\omega) \neq 0\}$. A functional $\Phi: L_{\rho}(X) \rightarrow \bar{R}$ is called to be additive if $\Phi(f+g)=\Phi(f)+\Phi(g)$, where the addition $\infty+(-\infty)$ is not permitted, for each $f, g \in L_{\rho}(X)$ such that $\mu(\operatorname{Supp} f \cap \operatorname{Supp} g)=0$. The additivity of $\Phi$ means that for each $f \in L_{\rho}(X)$ the set function $A \mapsto \Phi\left(1_{A} f\right)$ is finitely additive on $\mathcal{A}$. If $\Phi: L_{\rho}(X) \rightarrow \bar{R}$ is additive and proper, then $\Phi(0)=0$ is readily verified. The integral functional $I_{\phi}$ with $\phi(\omega, 0)=0$ a. e. is obviously additive on $L_{\rho}(X)$, if it is defined on $L_{\rho}(X)$. In the remainder of this section, we provide lemmas which will be needed in the next section.

LEMMA 4.1. If $\Phi: L_{\rho}(X) \rightarrow \bar{R}$ is an additive lower semicontinuous proper functional, then for each $f \in L_{\rho}(X)$ the set function $A \mapsto \Phi\left(1_{A} f\right)$ is countably additive on $\mathcal{A}$. 
Proof. Let $f \in L_{\rho}(X)$ and $A=\bigcup_{n=1}^{\infty} A_{n}$ with disjoint $A_{n} \in \mathcal{A}$. Then we have

$$
\Phi\left(1_{A} f\right)=\sum_{\imath=1}^{n} \Phi\left(1_{A_{\imath}} f\right)+\Phi\left(1_{B_{n}} f\right), \quad n \geqq 1,
$$

where $B_{n}=\bigcup_{i>n} A_{\imath}$. Since $\liminf _{n \rightarrow \infty} \Phi\left(1_{B_{n}} f\right) \geqq \Phi(0)=0$ by $\rho\left(1_{B_{n}}\|f\|\right) \downarrow 0$, it follows that

$$
\begin{aligned}
\Phi\left(1_{A} f\right) & \geqq \limsup _{n \rightarrow \infty} \sum_{\imath=1}^{n} \Phi\left(1_{A_{\imath}} f\right)+\liminf \Phi\left(1_{B_{n}} f\right) \\
& \geqq \operatorname{lim\operatorname {sup}} \sum_{n \rightarrow \infty}^{n} \Phi\left(1_{A_{\imath}} f\right) .
\end{aligned}
$$

On the other hand, since $\rho\left(\left\|\sum_{\imath=1}^{n} 1_{A_{\imath}} f-1_{A} f\right\|\right)=\rho\left(1_{B_{n}}\|f\|\right) \downarrow 0$, we have

$$
\Phi\left(1_{A} f\right) \leqq \liminf _{n \rightarrow \infty} \Phi\left(\sum_{\imath=1}^{n} 1_{A_{\imath}} f\right)=\liminf _{n \rightarrow \infty} \sum_{\imath=1}^{n} \Phi\left(1_{A_{\imath}} f\right) .
$$

Thus $\Phi\left(1_{A} f\right)=\sum_{\imath=1}^{\infty} \Phi\left(1_{A_{\imath}} f\right)$ is obtained.

The following three lemmas are concerned with the relationship between integral functionals and their kernel functions.

LEMMA 4.2. Let $\phi_{1}, \phi_{2}: \Omega \times X \rightarrow \bar{R}$ be two $\mathcal{A} \otimes \mathscr{B}_{X}$-measurable functions with $\phi_{1}(\omega, 0)=\phi_{2}(\omega, 0)=0$ a.e. such that $I_{\phi_{1}}(f) \leqq I_{\phi_{2}}(f)$ (resp. $\left.I_{\phi_{1}}(f)=I_{\phi_{2}}(f)\right)$ for each $f \in L_{\rho}(X)$ whenever both $I_{\phi_{1}}(f)$ and $I_{\phi_{2}}(f)$ are defined. Then there exists an $N \in \mathcal{A}$ with $\mu(N)=0$ such that $\phi_{1}(\omega, x) \leqq \phi_{2}(\omega, x)$ (resp. $\phi_{1}(\omega, x)=\phi_{2}(\omega, x)$ ) for all $\omega \in \Omega \backslash N$ and $x \in X$.

Proof. Taking Epi $\phi_{1}$, Epi $\phi_{2}: \Omega \rightarrow 2^{X \times R}$, we define $H: \Omega \rightarrow 2^{X \times R}$ by $H(\omega)$ $=\left(\right.$ Epi $\left.\phi_{2}\right)(\omega) \backslash\left(\right.$ Epi $\left.\phi_{1}\right)(\omega)$. Since $G\left(\right.$ Epi $\left.\phi_{1}\right), G\left(\right.$ Epi $\left.\phi_{2}\right) \in \mathcal{A} \otimes \mathscr{B}_{X \times R}$, it follows that $G(H)=G\left(\right.$ Epi $\left.\phi_{2}\right) \backslash G\left(\right.$ Epi $\left.\phi_{1}\right)$ is $\mathcal{A} \otimes \mathscr{B}_{X \times R}$-measurable. Thus it follows (cf. [17, Theorem 4]) that $D(H) \in \overline{\mathcal{A}}$. To prove the lemma, it suffices to show that $D(H)$ is $\mu$-null. Now suppose the contrary. By von Neumann-Aumann's selection theorem (cf. [9, Theorem 5.2], [17, Theorem 3]), there exists an $\bar{A}$ measurable function $(g, \zeta): \Omega \rightarrow X \times R$ such that $(g(\omega), \zeta(\omega)) \in H(\omega)$ for all $\omega \in D(H)$. Taking an $\mathcal{A}$-measurable function $(f, \xi): \Omega \rightarrow X \times R$ with $(f(\omega), \xi(\omega))$ $=(g(\omega), \zeta(\omega))$ a. e., we can choose an $A \in \mathcal{A}$ with $\mu(A)>0$ such that $(f(\omega), \xi(\omega))$ $\in H(\omega)$ for a. e. $\omega \in A$ and moreover $\left(1_{A} f, 1_{A} \xi\right) \in L_{\rho}(X) \times L_{1}$. Since $\phi_{1}(\omega, f(\omega))$ $>\xi(\omega) \geqq \phi_{2}(\omega, f(\omega))$ a. e. on $A$, it is seen that both $I_{\phi_{1}}\left(1_{A} f\right)$ and $I_{\phi_{2}}\left(1_{A} f\right)$ are defined, and hence we have

$$
I_{\dot{\delta}_{1}}\left(1_{A} f\right)=\int_{A} \phi_{1}(\omega, f(\omega)) d \mu>\int_{A} \xi d \mu
$$




$$
\geqq \int_{A} \phi_{2}(\omega, f(\omega)) d \mu=I_{\phi_{2}}\left(1_{A} f\right),
$$

a contradiction. This completes the proof.

LEMMA 4.3. Let $\phi: \Omega \times X \rightarrow \bar{R}$ be a normal function with $\phi(\omega, 0)=0$ a.e. such that $I_{\phi}$ is defined on $L_{\rho}(X)$. If $I_{\phi}$ is convex on $L_{\rho}(X)$, then $\phi(\omega, \cdot)$ is convex on $X$ for a.e. $\omega \in \Omega$.

Proof. Since $G($ Epi $\phi) \in \mathcal{A} \otimes \mathscr{B}_{X \times R}$ and $($ Epi $\phi)(\omega)$ is closed for every $\omega \in \Omega$, we can take, as observed in $\S 3$, an $F \in \mathscr{M}[\Omega ; X \times R]$ such that $F(\omega)=(\operatorname{Epi} \phi)(\omega)$ a. e. To prove the lemma, it suffices by Lemma 3.3 to show that $S_{\rho, 1}(F)$ is nonempty and convex. It is immediate that $(0,0) \in S_{\rho, 1}(F)$. The convexity assumption of $I_{\phi}$ means that Epi $I_{\phi}$ is convex in $L_{\rho}(X) \times R$. Thus the convexity of $S_{\rho, 1}(F)$ follows from the following observation: For each $(f, \xi) \in L_{\rho}(X) \times L_{1}$, $(f, \xi) \in S_{\rho, 1}(F)$ if and only if $\left(1_{A} f, \int_{A} \xi d \mu\right) \in \operatorname{Epi} I_{\phi}$ for all $A \in \mathcal{A}$. Indeed, $(f, \xi)$ $\in S_{\rho, 1}(F)$ if and only if $(f(\omega), \xi(\omega)) \in(\operatorname{Epi} \phi)(\omega)$ a. e., i. e., $\phi(\omega, f(\omega)) \leqq \xi(\omega)$ a. e. which is equivalent to $\int_{A} \phi(\omega, f(\omega)) d \mu \leqq \int_{A} \xi d \mu$ for all $A \in \mathcal{A}$. This means in view of $\phi(\omega, 0)=0$ a. e. that $\left(1_{A} f, \int_{A} \xi d \mu\right) \in \operatorname{Epi} I_{\phi}$ for all $A \in \mathcal{A}$. Thus the lemma is proved.

LEMMA 4.4. Let $\phi$ be as in Lemma 4.3. If there is an $\alpha \in R$ such that $I_{\phi}(f)$ $\geqq \alpha$ for all $f \in L_{\rho}(X)$, then there exists a $\xi \in L_{1}$ such that $\phi(\omega, x) \geqq \xi(\omega)$ on $X$ for a.e. $\omega \in \Omega$.

Proof. Take an $F \in \mathscr{M}[\Omega ; X \times R]$ as in the proof of Lemma 4.3. Since $(0,0) \in S_{\rho, 1}(F)$, there exists, by Lemma 3.2 , a sequence $\left\{\left(f_{n}, \xi_{n}\right)\right\}$ in $S_{\rho, 1}(F)$ such that $F(\omega)=\operatorname{cl}\left\{\left(f_{n}(\omega), \xi_{n}(\omega)\right)\right\}$ for all $\omega \in \Omega$. Then it is easy to see that

$$
\inf _{x \in X} \phi(\omega, x)=\inf _{n \geqq 1} \xi_{n}(\omega) \text { a. e. }
$$

Let $\zeta(\omega)=\inf _{n} \xi_{n}(\omega)$. Since $\zeta(\omega) \leqq \phi(\omega, 0)=0$ a. e., it now suffices to show that $\int_{\Omega} \zeta d \mu \geqq \alpha$. Suppose $\int_{\Omega} \zeta d \mu<\alpha$. Then a $\zeta^{\prime} \in L_{1}$ can be chosen so that $\zeta(\omega)<\zeta^{\prime}(\omega)$ a. e. and $\int_{\Omega} \zeta^{\prime} d \mu<\alpha$. It follows that there exists a countable measurable partition $\left\{A_{n}\right\}$ of $\Omega$ such that $\xi_{n}(\omega)<\zeta^{\prime}(\omega)$ a. e. on $A_{n}$ for $n \geqq 1$. Taking an integer $k$ such that $\int_{n=1}^{k} A_{n} \zeta^{\prime} d \mu<\alpha$ and defining $g=\sum_{n=1}^{k} 1_{A_{n}} f_{n} \in L_{\rho}(X)$, we have

$$
\begin{aligned}
I_{\phi}(g) & =\sum_{n=1}^{k} \int_{A_{n}} \phi\left(\omega, f_{n}(\omega)\right) d \mu \leqq \sum_{n=1}^{k} \int_{A_{n}} \xi_{n} d \mu \\
& \leqq \int_{n=1}^{k} \bigcup_{n=1}^{k} \zeta^{\prime} d \mu<\alpha,
\end{aligned}
$$


a contradiction, which completes the proof.

\section{§. Representation theorems.}

We now present integral representation theorems for several types of additive functionals on $L_{\rho}(X)$.

THEOREM 5.1. Let $\Phi: L_{\rho}(X) \rightarrow \bar{R}$ be an additive lower semicontinuous proper functional. Then there exists a normal function $\phi: \Omega \times X \rightarrow \bar{R}$ with $\phi(\omega, 0)=0$ a.e. such that $\phi(\omega, \cdot)$ is proper for every $\omega \in \Omega$ and $\Phi=I_{\phi}$ on $L_{\rho}(X)$. Moreover such a normal function $\phi$ is unique up to sets of the form $N \times X$ with $\mu(N)=0$.

Proof. The final uniqueness assertion follows immediately from Lemma 4.2. Since $\Phi$ is additive and proper, we get $\Phi(0)=0$. Define a subset $M$ of $L_{\rho}(X)$ $\times L_{1}$ by

$$
M=\left\{(f, \xi) \in L_{\rho}(X) \times L_{1}: \Phi\left(1_{A} f\right) \leqq \int_{A} \xi d \mu \text { for all } A \in \mathcal{A}\right\}
$$

Let $\left\{\left(f_{n}, \xi_{n}\right)\right\}$ be a sequence in $M$ convergent to $(f, \xi) \in L_{\rho}(X) \times L_{1}$. Then we have

$$
\Phi\left(1_{A} f\right) \leqq \liminf _{n \rightarrow \infty} \Phi\left(1_{A} f_{n}\right) \leqq \lim _{n \rightarrow \infty} \int_{A} \xi_{n} d \mu=\int_{A} \xi d \mu, \quad A \in \mathcal{A},
$$

and hence $(f, \xi) \in M$. Thus $M$ is closed in $L_{\rho}(X) \times L_{1}$. For each $(f, \xi),(g, \zeta)$ $\in M$ and $B \in \mathcal{A}$, we have

$$
\begin{aligned}
& \Phi\left(1_{A}\left(1_{B} f+1_{\Omega \backslash B} g\right)\right)=\Phi\left(1_{A \cap B} f\right)+\Phi\left(1_{A \backslash B} g\right) \\
& \quad \leqq \int_{A \cap B} \xi d \mu+\int_{A \backslash B} \zeta d \mu=\int_{A}\left(1_{B} \xi+1_{\Omega \backslash B} \zeta\right) d \mu, \quad A \in \mathcal{A},
\end{aligned}
$$

and hence $\left(1_{B} f+1_{\Omega \backslash B} g, 1_{B} \xi+1_{\Omega \backslash B} \zeta\right) \in M$. Thus $M$ is decomposable. Moreover $M$ is nonempty since $(0,0) \in M$. Thus, by Theorem 3.4 , there exists an $F \in$ $\mathscr{M}[\Omega ; X \times R]$ such that $M=S_{\rho, 1}(F)$. We can choose, by Lemma 3.2, a sequence $\left\{\left(f_{\imath}, \xi_{\imath}\right)\right\}$ in $S_{\rho, 1}(F)$ such that $F(\omega)=\operatorname{cl}\left\{\left(f_{i}(\omega), \xi_{i}(\omega)\right)\right\}$ for all $\omega \in \Omega$, and a sequence $\left\{\zeta_{j}\right\}$ in $L_{1}$ such that $\left\{\zeta_{j}(\omega)\right\}$ is dense in $[0, \infty)$ for every $\omega \in \Omega$. Since $\left(f_{\imath}, \xi_{\imath}+\zeta_{j}\right)$ $\in M$ for all $\imath, j \geqq 1$, we obtain

$$
F(\omega)=\operatorname{cl}\left\{\left(f_{i}(\omega), \xi_{\imath}(\omega)+\zeta_{j}(\omega)\right): \imath, j \geqq 1\right\} \text { a. e., }
$$

which shows that there exists an $N \in \mathcal{A}$ with $\mu(N)=0$ such that $(x, \alpha) \in F(\omega)$ implies $\{x\} \times[\alpha, \infty) \subset F(\omega)$ for each $\omega \in \Omega \backslash N$. Now define $\phi: \Omega \times X \rightarrow \bar{R}$ by

$$
\phi(\omega, x)= \begin{cases}\inf \{\alpha:(x, \alpha) \in F(\omega)\} & \text { if } \omega \in \Omega \backslash N \\ 0 & \text { if } \omega \in N .\end{cases}
$$

Then we have 


$$
(\operatorname{Epi} \phi)(\omega)=\left\{\begin{array}{lll}
F(\omega) & \text { if } & \omega \in \Omega \backslash N \\
X \times[0, \infty) & \text { if } \quad \omega \in N,
\end{array}\right.
$$

and hence Epi $\phi \in \mathscr{M}[\Omega ; X \times R]$ which implies that $\phi$ is normal. We shall then prove that $\Phi=I_{\phi}$ on $L_{\rho}(X)$ in the following three parts:

(I) Let $f \in L_{\rho}(X)$ and $\Phi(f)<\infty$. We show that $I_{\phi}(f)$ is defined and $I_{\phi}(f)$ $\leqq \Phi(f)$. In view of Lemma 4.1, the set function $A \mapsto \Phi\left(1_{A} f\right)$ is a $\mu$-continuous bounded signed measure on $\mathcal{A}$, and hence it has a Radon-Nikodym derivative $\xi \in L_{1}$ with respect to $\mu$. Then we have $(f, \xi) \in M$ and hence $(f(\omega), \xi(\omega)) \in F(\omega)$ a. e., so that $\phi(\omega, f(\omega)) \leqq \xi(\omega)$ a. e. This implies that $I_{\phi}(f)$ is defined and $I_{\phi}(f)$ $\leqq \int_{\Omega} \xi d \mu=\Phi(f)$.

(II) Let $f \in L_{\rho}(X)$ and assume that $I_{\phi}(f)$ is defined. We show that $\Phi(f)$ $\leqq I_{\phi}(f)$. Assuming $I_{\phi}(f)<\infty$, we can select a sequence $\left\{\xi_{n}\right\}$ in $L_{1}$ such that $\xi_{n}(\omega) \downarrow \phi(\omega, f(\omega))$ a. e. Since $\left(f(\omega), \xi_{n}(\omega)\right) \in($ Epi $\phi)(\omega)=F(\omega)$ a. e., we get $\left(f, \xi_{n}\right)$ $\in M$ for all $n$, and hence $\Phi(f) \leqq \int_{\Omega} \xi_{n} d \mu \downarrow I_{\phi}(f)$ by the monotone convergence theorem. Thus $\Phi(f) \leqq I_{\phi}(f)$.

(III) We now deduce that $I_{\phi}(f)$ is defined for every $f \in L_{\rho}(X)$. To see this, suppose that $I_{\phi}(f)$ is not defined, and let $A=\{\omega \in \Omega: \phi(\omega, f(\omega))<0\}$. Then it follows that $\int_{A} \phi(\omega, f(\omega)) d \mu=-\infty$. By part (I), we obtain $I_{\phi}(0) \leqq \Phi(0)=0$ and so $\int_{\Omega \backslash A} \phi(\omega, 0) d \mu<\infty$. Hence we have

$$
I_{\phi}\left(1_{A} f\right)=\int_{A} \phi(\omega, f(\omega)) d \mu+\int_{\Omega \backslash A} \phi(\omega, 0) d \mu=-\infty,
$$

so that by part (II) we have $\Phi\left(1_{A} f\right)=-\infty$ contradicting the assumption of $\Phi$ being proper.

The above three parts (I)-(III) yield that $\Phi=I_{\phi}$ on $L_{\rho}(X)$. We shall finally show that $\phi$ can be modified so as to satisfy the conditions in the theorem. Define $H: \Omega \rightarrow 2^{X}$ by $H(\omega)=\{x \in X: \phi(\omega, x)=-\infty\}$. Since $G(H) \in \mathcal{A} \otimes \mathscr{B}_{X}, D(H)$ $\in \overline{\bar{A}}$ and there exists an $\overline{\mathcal{A}}$-measurable function $g: \Omega \rightarrow X$ such that $g(\omega) \in H(\omega)$ for all $\omega \in D(H)$. Suppose that $D(H)$ is not $\mu$-null. Taking an $\mathcal{A}$-measurable function $f: \Omega \rightarrow X$ with $f(\omega)=g(\omega)$ a. e., we can choose an $A \in \mathcal{A}$ with $\mu(A)>0$ such that $f(\omega) \in H(\omega)$ for a. e. $\omega \in A$ and moreover $1_{A} f \in L_{\rho}(X)$. Then we have $\Phi\left(1_{A} f\right)=-\infty$, a contradiction, which implies that $D(H)$ is $\mu$-null. Since $\phi$ may be modified appropriately on a set $N \times X$ with $\mu(N)=0, \phi$ can be taken so that $\phi(\omega, \cdot)$ is proper for every $\omega \in \Omega$. Furthermore, in view of $\Phi(0)=0$, replacing $\phi(\omega, \cdot)$ by $\phi(\omega, \cdot)-\phi(\omega, 0)$ for $\omega \in \Omega$ with $\phi(\omega, 0)<\infty$, we can let $\phi(\omega, 0)=0$ a. e. Thus the proof is completed.

We call a function $\phi: \Omega \times X \rightarrow R$ to be of Carathéodory type if $\phi$ satisfies the following two conditions :

(i) $\phi(\cdot, x): \Omega \rightarrow R$ is measurable for each $x \in X$, 
(ii) $\phi(\omega, \cdot): X \rightarrow R$ is continuous for each $\omega \in \Omega$.

It is known (cf. [9, Theorem 6.1]) that a function of Carathéodory type as above is $\mathcal{A} \otimes \mathscr{B}_{X}$-measurable. In the usual definition of Carathéodory function, the condition (ii) is weakened so that $\phi(\omega, \cdot)$ is continuous for a. e. $\omega \in \Omega$. Whenever a function $\phi: \Omega \times X \rightarrow R$ is considered as an integral kernel function, we may modify $\phi$ appropriately on a set $N \times X$ with $\mu(N)=0$. Hence we adopt here the above definition. Let $\operatorname{Car}_{\rho}(\Omega ; X)$ denote the collection of all functions $\phi: \Phi \times X \rightarrow R$ of Carathéodory type such that for each $f \in L_{\rho}(X)$ the function $\phi(\omega, f(\omega))$ is in $L_{1}$.

THEOREM 5.2. If $\Phi: L_{\rho}(X) \rightarrow R$ is an additive contınuous functional, then there exists a $\phi \in \operatorname{Car}_{\rho}(\Omega ; X)$ with $\phi(\omega, 0)=0$ a.e. such that $\Phi=I_{\phi}$ on $L_{\rho}(X)$. Moreover such a function $\phi$ is unique up to sets of the form $N \times X$ with $\mu(N)=0$.

Proof. By Theorem 5.1, there exist two normal functions $\phi, \phi: \Omega \times X \rightarrow \bar{R}$ with $\phi(\omega, 0)=\phi(\omega, 0)=0$ a. e. such that $\Phi=I_{\phi}=-I_{\psi}$ on $L_{\rho}(X)$. Then, applying Lemma 4.2 , we can take an $N \in \mathcal{A}$ with $\mu(N)=0$ such that $\phi(\omega, x)=-\phi(\omega, x)$ for all $\omega \in \Omega \backslash N$ and $x \in X$. Redefining $\phi(\omega, x)=0$ on $N \times X$, we obtain a desired $\phi \in \operatorname{Car}_{\rho}(\Omega ; X)$.

REMARK. When $L_{\rho}(X)$ is a Banach space (for example, when $\rho$ has the weak Fatou property), it can be shown as in [10, pp. 22-25] that if $\phi$ $\in \operatorname{Car}_{\rho}(\Omega ; X)$, then the operator $T: L_{\rho}(X) \rightarrow L_{1}$ defined by $T f(\omega)=\phi(\omega, f(\omega))$ is continuous. Thus, in this situation, the converse of Theorem 5.2 holds: If $\phi \in \operatorname{Car}_{\rho}(\Omega ; X)$ and $\phi(\omega, 0)=0$ a. e., then the integral functional $I_{\phi}$ is additive and continuous on $L_{\rho}(X)$.

We denote by $\mathcal{L}_{\rho^{\prime}}\left(X^{*}\right)$ the space of all functions $f^{*}: \Omega \rightarrow X^{*}$ satisfying the following two conditions:

(1) $\left\langle x, f^{*}(\cdot)>: \Omega \rightarrow R\right.$ is measurable for each $x \in X$,

(2) the function $\left\|f^{*}\right\|=\left\|f^{*}(\cdot)\right\|$ is in $L_{\rho^{\prime}}$.

Note that the condition (1) implies the measurability of $\left\|f^{*}(\cdot)\right\|$. Under the usual identification of $\mu$-almost everywhere equal functions, $\mathcal{L}_{\rho^{\prime}}\left(X^{*}\right)$ is a normed linear space (in fact, a Banach space) with the norm $\rho^{\prime}\left(\left\|f^{*}\right\|\right)$.

THEOREM 5.3. The dual space $L_{\rho}(X)^{*}$ of $L_{\rho}(X)$ is isometrically isomorphic to $\mathcal{L}_{\rho^{\prime}}\left(X^{*}\right)$ under the bilinear form $\left\langle f, f^{*}\right\rangle=\int_{\Omega}\left\langle f(\omega), f^{*}(\omega)\right\rangle d \mu$ of $f \in L_{\rho}(X)$ and $f^{*} \in \mathcal{L}_{\rho^{\prime}}\left(X^{*}\right)$.

Proof. Let $f^{*} \in \mathcal{L}_{\rho^{\prime}}\left(X^{*}\right)$. For each $f \in L_{\rho}(X)$, it follows that the function $\left\langle f(\omega), f^{*}(\omega)\right\rangle$ is measurable and

$$
\int_{\Omega}\left|\left\langle f(\omega), f^{*}(\omega)\right\rangle\right| d \mu \leqq \int_{\Omega}\|f(\omega)\|\left\|f^{*}(\omega)\right\| d \mu \leqq \rho(\|f\|) \rho^{\prime}\left(\left\|f^{*}\right\|\right)<\infty .
$$

Thus the linear functional $\Phi(f)=\left\langle f, f^{*}\right\rangle$ is well-defined on $L_{\rho}(X)$ and we get $\|\Phi\| \leqq \rho^{\prime}\left(\left\|f^{*}\right\|\right)$. 
Conversely let $\Phi \in L_{\rho}(X)^{*}$. By Theorem 5.2, there exists a $\phi \in \operatorname{Car}_{\rho}(\Omega ; X)$ with $\phi(\omega, 0)=0$ a. e. such that $\Phi=I_{\phi}$ on $L_{\rho}(X)$. For each $f, g \in L_{\rho}(X)$ and each $\alpha, \beta \in R$, since

$$
\begin{aligned}
\int_{A} \phi(\omega, \alpha & f(\omega)+\beta g(\omega)) d \mu=\Phi\left(1_{A}(\alpha f+\beta g)\right) \\
& =\alpha \Phi\left(1_{A} f\right)+\beta \Phi\left(1_{A} g\right) \\
& =\int_{A}\{\alpha \phi(\omega, f(\omega))+\beta \phi(\omega, g(\omega))\} d \mu, \quad A \in \mathcal{A},
\end{aligned}
$$

it follows that $\phi(\omega, \alpha f(\omega)+\beta g(\omega))=\alpha \phi(\omega, f(\omega))+\beta \phi(\omega, g(\omega))$ a. e. There exists, as in Lemma 3.2, a sequence $\left\{f_{n}\right\}$ in $L_{\rho}(X)$ such that $\left\{f_{n}(\omega)\right\}$ is dense in $X$ for every $\omega \in \Omega$. We can now take an $N \in \mathcal{A}$ with $\mu(N)=0$ such that

$$
\phi\left(\omega, \alpha f_{i}(\omega)+\beta f_{\jmath}(\omega)\right)=\alpha \phi\left(\omega, f_{i}(\omega)\right)+\beta \phi\left(\omega, f_{j}(\omega)\right), \quad \omega \in \Omega \backslash N,
$$

for each $\imath, j \geqq 1$ and each rational numbers $\alpha, \beta$. This shows that $\phi(\omega, \cdot) \in X^{*}$ for every $\omega \in \Omega \backslash N$. Define

$$
f^{*}(\omega)=\left\{\begin{array}{lll}
\phi(\omega, \cdot) & \text { if } & \omega \in \Omega \backslash N \\
0 & \text { if } & \omega \in N .
\end{array}\right.
$$

Then it is clear that $f^{*}$ satisfies the above condition (1). It remains to show that $\rho^{\prime}\left(\left\|f^{*}\right\|\right) \leqq\|\Phi\|$. Since $\rho^{\prime}$ is a saturated function norm having the Fatou property, for any given $\varepsilon>0$ there exists a strictly positive $\eta \in M^{+}$with $\rho^{\prime}(\eta)$ $<\varepsilon$. Then we can select a measurable function $u: \Omega \rightarrow X$ such that $\|u(\omega)\| \leqq 1$ and $\left\langle u(\omega), f^{*}(\omega)\right\rangle \geqq \max \left(0,\left\|f^{*}(\omega)\right\|-\eta(\omega)\right)$ for all $\omega \in \Omega$. Putting $\zeta(\omega)=\langle u(\omega)$, $\left.f^{*}(\omega)\right\rangle$, we have $\zeta \in M^{+}$and $\left\|f^{*}\right\| \leqq \zeta+\eta$. For each $\xi \in M^{+}$with $\rho(\xi) \leqq 1$, it follows that

$$
\begin{aligned}
\int_{\Omega} \xi \zeta d \mu & =\int_{\Omega}\left\langle\xi(\omega) u(\omega), f^{*}(\omega)\right\rangle d \mu \\
& =\Phi(\xi u) \leqq\|\Phi\| \rho(\|\xi u\|) \leqq\|\Phi\|,
\end{aligned}
$$

which shows $\rho^{\prime}(\zeta) \leqq\|\Phi\|$ and so $\rho^{\prime}\left(\left\|f^{*}\right\|\right) \leqq \rho^{\prime}(\zeta)+\rho^{\prime}(\eta)<\|\Phi\|+\varepsilon$. Thus we have the desired conclusion.

REMARK 1 . When $\rho$ is not necessarily absolutely continuous, Theorem 5.3 is extended as follows: If the carrier of $L_{\rho}^{a}$ is the whole set $\Omega$, then $L_{\rho}^{a}(X)^{*}$ is isometrically isomorphic to $\mathcal{L}_{\rho^{\prime}}\left(X^{*}\right)$ in the manner as in Theorem 5.3.

REMARK 2. If $X^{*}$ is separable, or equivalently if $X^{*}$ has the Radon-Nikodym property (cf. [18]), then Theorem 5.3 asserts that $L_{\rho}(X)^{*}$ is isometrically isomorphic to $L_{\rho^{\prime}}\left(X^{*}\right)$. This conclusion is a special case of [7, Theorem 3.2], but $\rho$ is as- sumed in [7] to have the weak Fatou property.

For the case of lower semicontinuous convex functionals, we give a representation theorem in a somewhat detailed form.

THEOREM 5.4. For each proper functional $\Phi: L_{\rho}(X) \rightarrow \bar{R}, \Phi$ is additive lower 
semicontinuous and convex if and only if there exists a normal function $\phi: \Omega \times$ $X \rightarrow \bar{R}$ with $\phi(\omega, 0)=0$ a.e. such that

(i) $\phi(\omega, \cdot)$ is proper and convex for every $\omega \in \Omega$,

(ii) there exists an $f^{*} \in \mathcal{L}_{\rho^{\prime}}\left(X^{*}\right)$ and a $\xi \in L_{1}$ satisfying $\phi(\omega, x) \geqq\left\langle x, f^{*}(\omega)\right\rangle$ $+\xi(\omega)$ on $X$ for a.e. $\omega \in \Omega$,

(iii) $\Phi=I_{\phi}$ on $L_{\rho}(X)$.

Proof. Let $\Phi: L_{\rho}(X) \rightarrow \bar{R}$ be additive, lower semicontinuous, proper, and convex. By Theorem 5.1 and Lemma 4.3, there exists a normal function $\phi: \Omega \times X \rightarrow \bar{R}$ with $\phi(\omega, 0)=0$ a. e. for which the conditions (i) and (iii) are satisfied. Since Epi $\Phi$ is closed and convex in $L_{\rho}(X) \times R$ and $(0,-1) \notin$ Epi $\Phi$, the separation theorem gives, in view of Theorem 5.3, an $f^{*} \in \mathcal{L}_{\rho^{\prime}}\left(X^{*}\right)$ and a $\beta \in R$ such that $\left\langle f, f^{*}\right\rangle+\alpha \beta<-\beta$ for all $(f, \alpha) \in$ Epi $\Phi$. Then $\beta<0$ follows from $(0,0) \in \operatorname{Epi} \Phi$, and hence we can let $\beta=-1$. We now have

$$
\begin{aligned}
\int_{\Omega}\{\phi(\omega, & \left.f(\omega))-\left\langle f(\omega), f^{*}(\omega)\right\rangle\right\} d \mu \\
= &
\end{aligned}
$$

which implies the condition (ii) by Lemma 4.4 .

Conversely let $\phi$ be a normal function with $\phi(\omega, 0)=0$ a. e. satisfying (i)(iii). It is immediate that $\Phi=I_{\phi}$ is additive and convex. To show the lower semicontinuity, let $\left\{f_{n}\right\} \subset L_{\rho}(X), f \in L_{\rho}(X)$, and $\rho\left(\left\|f_{n}-f\right\|\right) \rightarrow 0$. As is seen from the proof of Lemma 3.1, we can select a subsequence $\left\{g_{k}\right\}$ of $\left\{f_{n}\right\}$ such that $\left\|g_{k}(\omega)-f(\omega)\right\| \rightarrow 0$ a. e. and $\Phi\left(g_{k}\right) \rightarrow \lim _{n \rightarrow \infty} \inf \Phi\left(f_{n}\right)$. Then, using Fatou's lemma, we have

$$
\begin{aligned}
\Phi(f)- & \left\langle f, f^{*}\right\rangle-\int_{\Omega} \xi d \mu \\
& =\int_{\Omega}\left\{\phi(\omega, f(\omega))-\left\langle f(\omega), f^{*}(\omega)\right\rangle-\xi(\omega)\right\} d \mu \\
& \leqq \int_{\Omega} \liminf _{k \rightarrow \infty}\left\{\phi\left(\omega, g_{k}(\omega)\right)-\left\langle g_{k}(\omega), f^{*}(\omega)\right\rangle-\xi(\omega)\right\} d \mu \\
& \leqq \lim _{k \rightarrow \infty}\left\{\Phi\left(g_{k}\right)-\left\langle g_{k}, f^{*}\right\rangle-\int_{\Omega} \xi d \mu\right\} \\
& =\liminf _{n \rightarrow \infty} \Phi\left(f_{n}\right)-\left\langle f, f^{*}\right\rangle-\int_{\Omega} \xi d \mu,
\end{aligned}
$$

and hence $\Phi(f) \leqq \liminf _{n \rightarrow \infty} \Phi\left(f_{n}\right)$. The proof is now completed.

\section{REFERENCES}

[1] R. A. Alò AND A. DE KORVIN, Representation of Hammerstein operators by Nemytskii measures, J. Math. Anal. Appl., 52 (1975), 490-513.

[2] J. BAtT, Nonlinear integral operators on $C(S, E)$, Studia Math., 48 (1973), 
145-177.

[3] L. Drewnowski and W. Orlicz, On orthogonally additive functionals, Bull. Acad. Polon. Sci. Sér. Sci. Math. Astronom. Phys., 16 (1968), 883-888.

[4] L. DREWNowski AND W. ORlicz, On representation of orthogonally additive functionals, Bull. Acad. Polon. Sc1. Sér. Sc1. Math. Astronom. Phys., 17 (1969), 167-173.

[5] L. DREWNowski AND W. ORLICZ, Continuity and representation of orthogonally additive functionals, Bull. Acad. Polon. Sci. Sér. Scı. Math. Astronom. Phys., 17 (1969), 647-653.

[6] N. A. Friedman and M. Kat $Z$, Additive functionals on $L_{p}$ spaces, Canad. J. Math., 18 (1966), 1264-1271.

[7] N.E. Gretsky and J.J. Uhl, JR., Bounded linear operators on Banach function spaces of vector-valued functions, Trans. Amer. Math. Soc., 167 (1972), 263-277.

[8] F. HIAI AND H. UMEGAKI, Integrals, conditional expectations, and martingales of multivalued functions, J. Multivariate Anal., 7 (1977), 149-182.

[9] C. J. Himmelberg, Measurable relations, Fund. Math., 87 (1975), 53-72.

[10] M. A. KRasnosel'skil, Topological Methods in the Theory of Nonlinear Integral Equations, translated by J. Burlak, Macmillan, New York, 1964.

[11] A. D. Martin AND V. J. Mizel, A representation theorem for certain nonlinear functionals, Arch. Rational Mech. Anal., 15 (1964), 353-367.

[12] V. J. Mizel, Characterization of non-linear transformations possessing kernels, Canad. J. Math., 22 (1970), 449-471.

[13] V.J. Mizel and K. Sundaresan, Representation of additive and biadditive functionals, Arch. Rational Mech. Anal., 30 (1968), 102-126.

[14] V.J. Mizel and K. Sundaresan, Additive functionals on spaces with nonabsolutely-continuous norm, Bull. Acad. Polon. Sci. Sér. Sc1. Math. Astronom. Phys., 18 (1970), 385-389.

[15] V.J. Mizel and K. Sundaresan, Representation of vector valued nonlinear functions, Trans. Amer. Math. Soc., 159 (1971), 111-127.

[16] J.A. Palagallo, A representation of additive functionals on $L^{p}$-spaces, $0<p<1$, Pacific J. Math., 66 (1976), 221-234.

[17] M.-F. Sainte-Beuve, On the extension of von Neumann-Aumann's theorem, J. Functional Anal., 17 (1974), 112-129.

[18] C. Stegall, The Radon-Nikodym property in conjugate Banach spaces, Trans. Amer. Math. Soc., 206 (1975), 213-223.

[19] K. Sundaresan, Additive functionals on Orlicz spaces, Studia Math., 32 (1969), 269-276.

[20] D. H. WAGNer, Survey of measurable selection theorems, SIAM J. Control and Optımization, 15 (1977), 859-903.

[21] W. A. WoyczyŃski, Additive functionals on Orlicz spaces, Colloq. Math., 19 (1968), 319-326.

[22] A.C. ZAANEN, Integration, revised ed., North-Holland, Amsterdam, 1967.

Department of Information Sciences

SCIENCE UNIVERSITY OF TOKYO

Noda City, Chiba 278, JaPan 